\title{
Study on Formation Mechanism of Zonal Disintegration of Surrounding Rock in Deep Tunnel
}

\author{
Heng Zhou ${ }^{1,2 *}$, Xiaofan $\mathrm{An}^{1,2}$, Ying Zhang ${ }^{1,2}$, Xuguang $\mathrm{Chen}^{3}$, Shengjie Di ${ }^{1,2}, \mathrm{Xi} \mathrm{Lu}^{1,2}$ \\ ${ }^{1}$ Power China, Northwest Engineering Corporation Limited, $710065 \mathrm{Xi}$ 'an, Shaanxi, China \\ ${ }^{2}$ High Slope and Geological Hazard Research \& Management Branch, National Energy and Hydropower Engineering Technology R\&D \\ Centre, 710065 Xi'an, Shaanxi, China \\ ${ }^{3}$ Ocean University of China, 266100, Qingdao, Shandong, China
}

\begin{abstract}
With the construction of underground rock engineering, the surrounding rock in deep tunnels appears zonal disintegration of fracture and intact zones alternate distribution, which is a special engineering geological phenomenon. This study establishes a partitioned fracture model under the coupling of high in-situ stress and osmotic pressure, and identify the key influencing factors of the fracture model. Furthermore, a stress intensity factor (SIF) of initial cracks on surrounding rock elastoplastic boundary is derived using the transformation of complex functions. Considering the high seepage pressure of the surrounding rocks, a zonal fracture initiation criterion is established combined with the analysis of redistributed stress fields. Finally, the obtained criterion is embedded into an extended finite element method (XFEM) platform for numerical simulation. Taking the maximum circumferential tensile stress as a cracking criterion, the propagation trajectory of rock cracks is traced by contour methods. Calculation results have realized the modelling of a whole process of crack initiation, propagation, and formation. And the established criterion has be verified.
\end{abstract}

\section{Introduction}

Deep rock mechanics has become a hot research area in the field of rock mechanics because it meets the needs of a series of new scientific phenomena such as the deformation and failure of surrounding rocks in deep rock engineering. In addition to rock bursts and large deformations of surrounding rocks, the regional fracture of surrounding rocks also attracts the attention of many researchers. Zonal disintegration refers to the phenomenon of alternating fractured and non-fractured areas in surrounding rocks during the excavation of tunnels in deep rock mass [1].

Adams and Jager [2], Shemyakin et al. [3] found zonal disintegration in deep mining sites in South Africa and the former Soviet Union, respectively. China's Jinping II hydropower station also found related phenomena of zonal rupture, such as the pie-shaped cores and intact cores alternately distributed in the $3 \#$ adit, and acoustic monitoring data of the loose circle of auxiliary tunnels showed that it contains several rupture and non-rupture zones [4]. Shemyakin (1986) first verified the zonal disintegration found in Taimyrskii's deep mining site through model tests [5].

Although on-site and experimental monitoring has confirmed the existence of zonal disintegration, further understanding of evolution features and the structural effects of surrounding rock after zonal rupture is needed. In terms of the causes and formations of zone disintegration, Zhou and Qian [6] used fracture mechanics theory. He [7] adopted the approach of energy balance analysis, and Chen [8] used elastoplastic theory to study the formation conditions of zone disintegration. Although scholars have adopted as many as a dozen theoretical models, they have not yet formed a generally accepted and satisfactory explanation for its formation mechanism and conditions at present.

In terms of zonal fracture evolution mode, fracture shape and rock deformation law, Kurlenya and Oparin [9] gave an empirical formula for the radius and thickness of each fracture zone. Guzev [10] used a non-Euclidean geometric model to study the reasons for the periodic changes in the shape (distribution range) of fracture zones with the stress of surrounding rocks. Metolov [11] believes that parameter weakening caused by unloading of surrounding rocks is the reason for the non-monotonic change of stress in zonal fracture. In general, the evolution of zonal rupture and the derivation sequence of each rupture circle need to be further clarified, and the main controlling factors affecting the rupture evolution mode need to be identified.

In this study, a partitioned fracture evolution model under the coupling of high in-situ stress and osmotic pressure is established, and the key influencing factors of the fracture mode are identified. The SIF of initial cracks at the elastoplastic boundary of surrounding rocks is derived based on the transformation of complex functions. Considering the high seepage pressure of

\footnotetext{
* Corresponding author: 1728@nwh.cn
} 
surrounding rocks, the initiation criterion for zonal fracture is established combined with the analysis of redistributed stress fields. Furthermore, the obtained criterion is embedded in an XFEM platform for numerical simulation. Taking the maximum circumferential tensile stress criterion as a cracking criterion, the propagation trajectory of cracks is traced through contour methods, which realized the modelling of the whole process of crack initiation, propagation, and formation, and verified the established criterion.

\section{Crack propagation trajectory of rock under compression}

Rock cracks will expand and propagate under compression. The initial direction of cracks is not consistent with original cracks, but deflected by a large angle. Tests or numerical modeling confirm that the crack initiation angle is $70.5^{\circ}$, and the growth trajectory after cracking is not a straight line. Horri and Nemat [15] used complex functions to calculate the crack growth trajectory by adopting the crack growth criterion $K_{I}=K_{k}$. Calculations show that the crack propagation angle $\theta$ gradually deviates from the direction of the maximum principal stress $\sigma 1$ and is eventually parallel to it. However, the formula used in the calculation of crack growth is a multi-order complex singular integral equation, so a closed solution cannot be obtained, and it is difficult to use numerical methods. Some scholars have simplified and approximated it, but these approximations are far from the actual situation. Therefore, this study uses the following numerical method as shown in the initial crack in Fig. 1 to calculate the crack propagation direction.

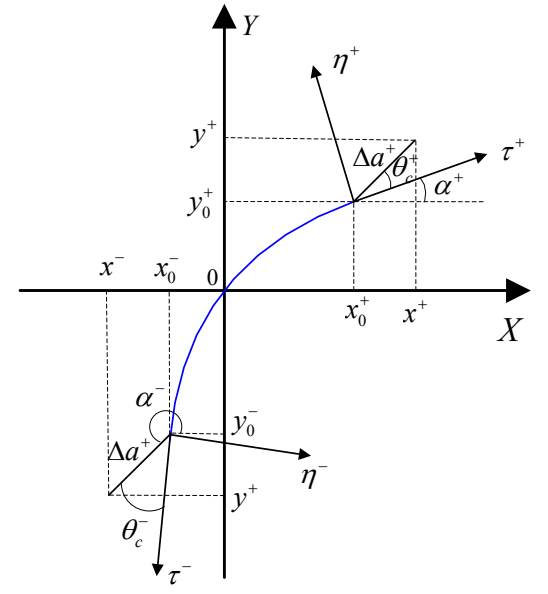

Fig. 1. Model of crack growth trajectory

$$
L_{0}=\left\{x_{0}(t)+l \cdot y_{0}(t)\right\}
$$

Define $\left[x_{0}^{ \pm}=x_{0}( \pm 1), y_{0}^{ \pm}=y_{0}( \pm 1)\right]$ as the coordinate value of initial crack tip; define $\theta_{c}^{ \pm}$as the initial crack angle, which is determined by the cracking criterion. Taking the maximum circumferential stress criterion,

$$
K_{I} \sin \theta_{c}^{ \pm}+K_{I I}\left(3 \cos \theta_{c}^{ \pm}-1\right)=0
$$

$$
\theta_{c}^{ \pm}=\arccos \frac{3 K_{I I}^{2}+\sqrt{K_{I}^{2}+8 K_{I}^{2} K_{I I}^{2}}}{K_{I}^{2}+9 K_{I I}^{2}}
$$

After determining the cracking direction angle, the maximum circumferential stress at the crack tip can be expressed as,

$$
\left(\sigma_{\theta}\right)_{\max }=\frac{1}{\sqrt{2 \pi r_{0}}} \cos \frac{\theta_{c}^{ \pm}}{2}\left[K_{I} \cos ^{2} \frac{\theta_{c}^{ \pm}}{2}-\frac{3}{2} K_{I I} \sin \theta_{c}^{ \pm}\right]
$$

The maximum circumferential stress criterion is used to determine whether the crack continues to grow. When the following formula is satisfied, the crack begins to grow,

$$
\left(\sigma_{\theta}\right)_{c}=\frac{K_{I c}}{\sqrt{2 \pi r_{0}}}
$$

Substituting Eq. (5) into Eq. (4), the condition of crack growth can be obtained as,

$$
\cos \frac{\theta_{c}^{ \pm}}{2}\left[K_{I} \cos ^{2} \frac{\theta_{c}^{ \pm}}{2}-\frac{3}{2} K_{I I} \sin \theta_{c}^{ \pm}\right]=K_{I c}
$$

Let $\Delta a^{ \pm}$be the increment of crack propagation in one step, which is determined by the crack propagation criterion. The coordinate value of crack tip newly generated after one step of propagation can be calculated by Eq. (7),

$$
\left[\begin{array}{l}
x^{ \pm}=x_{0}( \pm 1)+\Delta a^{ \pm} \cdot \cos \left(\theta_{c}^{ \pm}+\alpha^{ \pm}\right) \\
y^{ \pm}=y_{0}( \pm 1)+\Delta a^{ \pm} \cdot \cos \left(\theta_{c}^{ \pm}+\alpha^{ \pm}\right)
\end{array}\right]
$$

where $\Delta a^{ \pm}$is calculated by the following equation.

$$
a^{ \pm}=\left\{\begin{array}{c}
\frac{d y( \pm 1)}{d t} ; \pm \frac{d x( \pm 1)}{d t}>0 \\
\arctan \frac{d x( \pm 1)}{d t} \\
\pi+\arctan \frac{\frac{d y( \pm 1)}{d t}}{\frac{d x( \pm 1)}{d t}} ; \pm \frac{d x( \pm 1)}{d t}<0
\end{array}\right\}
$$

Polynomial fitting can be used to obtain the contour of the crack after an incremental step $\Delta a^{ \pm}$is extended,

$$
L_{1}=\left\{x_{1}(t)+l \cdot y_{1}(t)\right\}, \quad|t| \leq 1
$$

Let the variable $s$ denote the arc length of a crack curve,

$$
s(t)=\int_{-1}^{t} \sqrt{\left(\frac{d x_{0}}{d t}\right)^{2}+\left(\frac{d y_{0}}{d t}\right)^{2}} d t
$$

where $0 \leq s(t) \leq\left|L_{0}\right|,\left|L_{0}\right|$ is the length of initial cracks.

Then generate the contour point matrix of initial cracks,

$$
\left[\begin{array}{l}
X_{0}^{T}=\left(x_{01}, x_{02}, \cdots, x_{0 n}\right) \\
Y_{0}^{T}=\left(y_{01}, y_{02}, \cdots, y_{0 n}\right) \\
s_{0}^{T}=\left(0, s_{02}, \cdots, s_{0 n-1}, s_{0 n}\right)
\end{array}\right]
$$

where $x_{0 i}=x_{0}\left(t_{i}\right), y_{0 i}=y_{0}\left(t_{i}\right), s_{0 i}=s_{0}\left(t_{i}\right)$. To determine the value of $t$, the arc length of each adjacent point must be consistent.

The initial crack angle can be, 
The coordinate of a new crack tip is $\left(x_{1}^{ \pm}, y_{1}^{ \pm}\right)$after the crack's propagation length $\Delta a^{ \pm}$along the direction of $\theta^{* \pm}$. Then a matrix of new crack contours after propagation forms as,

$$
X_{1}=\left\{\begin{array}{l}
x_{1}^{-} \\
\left\{X_{0}\right\} \\
x_{1}^{+}
\end{array}\right\} ; \quad Y_{1}=\left\{\begin{array}{l}
y_{1}^{-} \\
\left\{Y_{0}\right\} \\
y_{1}^{+}
\end{array}\right\} ; \quad s_{1}=\left\{\begin{array}{l}
0 \\
\left\{s_{0}\right\}+\Delta a^{-} \\
\left|L_{0}\right|+\Delta a^{+}+\Delta a^{-}
\end{array}\right.
$$

Curve $x_{1}\left(s_{1}\right), y_{1}\left(s_{1}\right)\left(0 \leq s_{1} \leq\left|L_{1}\right|\right)$ can be generated through a set of specific points, which approximates the new contour of crack $L_{1}$. Define $L_{1}$ as the length of a new crack, input new variables to parameterize crack profile,

$$
S_{1}(t)=\frac{1+t}{2} \cdot\left|L_{1}\right| ;|t| \leq 1
$$

Eq. (12) satisfies: $s_{1}(-1)=0, s_{1}(1)=\left|L_{1}\right|$. Therefore, the crack function is established as,

$$
L_{1}=\left\{x_{1}(t)+l \cdot y_{1}(t)\right\} ;|t| \leq 1
$$

Repeat this process for each incremental step to obtain the parameter equation of the crack profile. Then the intensity factor of the crack tip is calculated, and the propagation direction of the crack and the coordinates of the newly generated crack tip are obtained.

Mechanical parameters of the on-site rock samples from Huainan Coal Mine are used as the basis for calculation. Its physical and mechanical indexes are shown in Table 1: $E=12.97 \mathrm{GPa}, C=12 \mathrm{MPa}, \mu=0.268$, $K_{I C}=2.19 \mathrm{MPa} \cdot \mathrm{m}^{1 / 2}$.

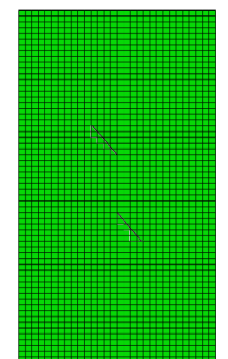

Fig. 2. Numerical model of crack propagation under uniaxial compression

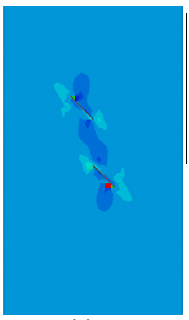

(a)

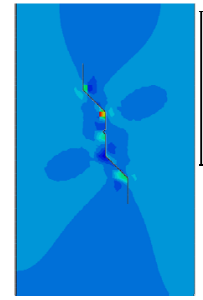

(d)

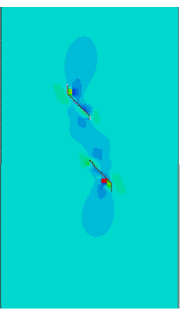

(b)

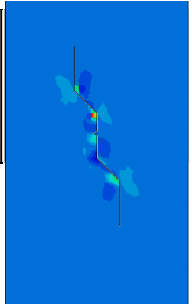

(e)

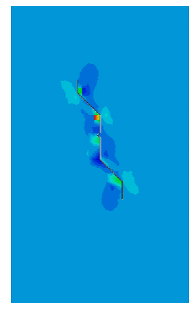

(c)

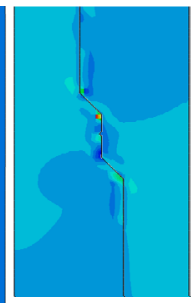

(f)
Fig. 3. Numerical simulation results of crack growth
Fig. 3 shows the crack propagation process under uniaxial compressive load. Numerical simulation reveals that the crack propagation under compression gradually deviates to the direction of the maximum principal stress, until it is parallel to the maximum principal stress. This phenomenon can explain the formation mechanism of splitting failure of surrounding rocks at the sidewalls of tunnels.

\section{Stress characteristics of surrounding rock during zonal disintegration}

Taking into account the complexity of actual projects, mechanical analysis is carried out after simplifying a deep tunnel. Assuming that the tunnel is a circle with an excavation radius of $r_{a}$, under the state of hydrostatic pressure $P_{0}$, the lateral pressure coefficient $\lambda$ is 1.0.

After the excavation and unloading of a tunnel, surrounding rocks will produce stress redistribution under high in-situ stress. The surrounding rocks enter a plastic stage when the internal stress increases large enough to exceed the yield limit of the rocks. Using Mohr-Coulomb yield criterion $\sin \phi=\frac{\sigma_{\theta}-\sigma_{r}}{\sigma_{\theta}+\sigma_{r}+2 c \cot \phi}$ and related knowledge of elastoplastic mechanics, the Kastner formula can be obtained,

$$
R_{p}=r_{a}\left[\frac{\left(p_{0}+c \cot \phi\right)(1-\sin \phi)}{c \cot \phi}\right]^{\frac{1-\sin \phi}{2 \sin \phi}}
$$

The expressions of stress in the plastic zone and elastic zone are respectively,

$$
\left.\begin{array}{c}
\sigma_{r p}=c \cot \phi\left(\frac{r}{r_{a}}\right)^{\frac{2 \sin \phi}{1-\sin \phi}-c \cot \phi} \\
\sigma_{\theta p}=\frac{1+\sin \phi}{1-\sin \phi} c \cot \phi\left(\frac{r}{r_{a}}\right)^{\frac{2 \sin \phi}{1-\sin \phi}}-c \cot \phi \\
\sigma_{r e}=p_{0}-\left(p_{0} \sin \phi+c \cos \phi\right) \frac{R_{p}^{2}}{r^{2}} \\
\sigma_{\theta e}=p_{0}+\left(p_{0} \sin \phi+c \cos \phi\right) \frac{R_{p}^{2}}{r^{2}}
\end{array}\right\}
$$

where $\sigma_{r}$ is the radial stress of surrounding rocks; $\sigma_{\theta}$ is the radial stress of surrounding rocks; $c$ and $\phi$ are the cohesion and friction coefficient of surrounding rocks; $R_{p}$ is the radius of the plastic zone; $r$ is the radius of a tunnel.

Fig. 4 exhibits the elastoplastic stress distribution of surrounding rocks of a circular tunnel.

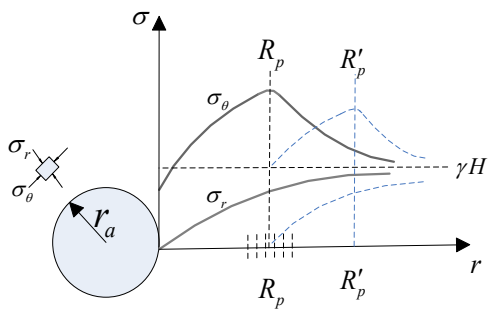

Fig. 4. Elastoplastic stress field of surrounding rock of circular tunnel 
It can be seen from Fig. 5 that the radial stress $\sigma_{r}$ of surrounding rocks is the minimum principal stress; the tangential stress $\sigma_{\theta}$ is the maximum principal stress, and its peak appears at the elastoplastic boundary. Therefore, the crack of surrounding rocks at the boundary will first meet its failure conditions to rupture and propagate. Calculation shows that the crack will propagate along the direction of the maximum principal stress (that is, the direction of the tangential stress $\sigma_{\theta}$ ), and evolve until it penetrates, forming a ring-shaped fracture concentric with the excavated cavity at the elastoplastic boundary.

After the ring-shaped fracture forms, it is equivalent to excavating a ring-shaped surrounding rock with a thickness of $r_{a}<r<R_{p}$, which will cause unexcavated rocks to unload and generate a new stress redistribution. If the internal stress of surrounding rocks near the newly excavated unloading surface $\left(r=R_{p}\right)$ still satisfies the Mohr-Coulomb criterion, a new boundary between the plastic zone and elastoplastic zone in surrounding rocks will generate. The stress state on the boundary of the new plastic zone is similar to that shown in Fig. 5. If the crack initiation conditions are met, the above process will be repeated, resulting in the formation of a second ring-shaped fracture. Similar to the foregoing, the fracture is still concentric with the excavation tunnel. The continuous cycle and progress of this process led to the appearance of zonal disintegration.

\section{Conclusion}

(1) The initiation criterion of arc cracks in surrounding rocks of tunnels is established. A slip crack model in a polar coordinate system is proposed, and then it is equivalent to an arc crack according to propagation characteristics. The SIF of cracks for circular tunnels is calculated by the complex function, and it is believed that the ring-shaped fracture begins to form when it is greater than the fracture toughness of surrounding rocks.

(2) A simplified calculation method for crack propagation under compression is proposed. Through the study of the secondary stress field of surrounding rocks of deep tunnels, the formation mechanism of zonal disintegration is revealed. That is, there is an incompatibility between the peak value of tangential stress and the radial strain at the boundary of the plastic zone of surrounding rocks, which causes the formation of a ring-shaped fracture concentric with the excavated tunnel.

(3) Numerical modeling of the entire process of zonal rupture initiation, expansion, and formation is realized based on the XFEM. The established criterion is verified.

(4) The mechanism of zonal disintegration is: under high in-situ stress, the cracks at the boundary of the plastic zone of surrounding rocks first meet the fracture criterion and then rupture and expand along the tangential direction. When the released strain energy of surrounding rocks is greater than the required surface energy, a ring-shaped fracture will form. As the plastic zone advances to the deep, the process is continuously cycled to form a unique phenomenon of high in-situ stress surrounding rocks.

\section{References}

1. Q. Qian. (2004) The current development of nonlinear rock mechanics: the mechanics problems of deep rock mass. In: Proceedings of the 8th National Conference on Rock Mechanics and Engineering. Beijing.

2. G.D. Adam, A.J. Jager.(1980)Etroscopic observations of rock fracturing ahead of faces in deep-level gold mines. J. S. Afr. I. Min. Metall. 2.

3. E.I. Shemyakin, G.L. Fisenko, M.V. Kurlenya, et al(1986). Zonal disintegration of rocks around underground workings, part I: data of in-situ observations. J. Min. Sci. 22, 3.

4. N. Liu, C. Zhang, W. Chu.(2012)Fracture Characteristics and damage evolution lae of Jinping deep marble. Chin. J. Rock Mech. Eng. 31, 8

5. E.I. Shemyakin, G.L. Fisenko, M.V. Kurlenya, et al. (1986) Zonal disintegration of rocks around underground workings, part II: rock fracture simulated in equivalent materials. J. Min. Sci. 22, 4

6. X. Zhou, Q. Qian. (2007)Zonal fracturing mechanism in deep tunnel. Chin. J. Rock Mech. Eng. 26, 5 .

7. Y. He, B. Jiang, L. Han, et al. (2008)Study of intermittent zonal fracturing of surrounding rock in deep roadways. J. China Uni. Min. Tech. 37, 3.

8. J. Chen, C. Zhu, C. Zhu, et al. (2010) Elasticplastic-brittle analysis of zonal disintegration within rock mass in deep tunnel. J. China Coal Soc. 35, 4.

9. M.V. Kurlenya, V.N.Oparin. (1996)Scale factor of phenomen- on of zonal disintegration of rock, and canonical series of atomic and ionic radii. J. Min. Sci. 32, 2.

10. M.A. Guzev, A. A. (2001) Paroshin. Non-euclidean model of the zonal disintegration of rocks around an underground working. J Appl. Mech. Tech. Phys. 42, 1.

11. L.S. Metolov, A.F. Morozov. (2002) Physical foundations of mechanism of zonal rock failure in the vincity of mine working. J. Min. Sci. 38, 2.

12. H. Horii, S. Nemat-nasser. (1985) Compressioninduced microcrack growth in brittle solid: axial splitting and shear failure. J. Geophys. Res. 90. 